\title{
Left Ventricular Diastolic Function in Type 2 Diabetes Mellitus and the Association With Coronary Artery Calcium Score: A Cardiac MRI Study
}

\section{Bruno Graça ${ }^{1,2}$ \\ Paulo Donato ${ }^{1,2}$ \\ Maria João Ferreira ${ }^{1,3}$ \\ Miguel Castelo-Branco ${ }^{1}$ \\ Filipe Caseiro-Alves ${ }^{1,2}$}

Keywords: cardiac imaging techniques, diastolic function, MRI, peak filling rate, transmitral flow

\section{DOI:10.2214/AJR.13.11325}

Received May 28, 2013; accepted after revision August 27, 2013 .

This research was financially supported by Project "DolT," which is cofinanced by the European Community Fund FEDER through COMPETE.

${ }^{1}$ Faculty of Medicine, University of Coimbra, Azinhaga de Santa Comba, 3000-548, Coimbra, Portugal. Address correspondence to B. Graça (brunomgraca@gmail.com).

${ }^{2}$ Medical Imaging Department, Coimbra's Hospital Centre and University, Coimbra, Portugal.

${ }^{3}$ Cardiology Department, Coimbra's Hospital Centre and University, Coimbra, Portugal.

This article is available for credit.

AJR2014; 202:1207-1214

0361-803X/14/2026-1207

(c) American Roentgen Ray Society

OBJECTIVE. The purpose of this study was to compare cardiac MRI-derived parameters of left ventricular (LV) diastolic function between uncomplicated type 2 diabetes mellitus (DM2) and normoglycemic control subjects and to evaluate whether these parameters of LV diastolic function are related to coronary atherosclerosis.

SUBJECTS AND METHODS. We prospectively studied 41 subjects with DM2 and 21 normoglycemic control subjects (30 women and 32 men; mean age, $57.2 \pm 7.1$ [SD] years) with no evidence of overt cardiovascular disease. We used cardiac MRI to measure LV volumes, LV peak filling rate (PFR), and transmitral flow and CT to determine coronary artery calcium scores.

RESULTS. Absolute values of the peak filling rate (PFR) were significantly lower in DM2 patients than in control subjects (mean \pm SD, $293.2 \pm 51.7$ vs $375.7 \pm 102.8 \mathrm{~mL} / \mathrm{s}$, respectively; $p<0.001$ ). Mitral peak E velocities (mean $\pm \mathrm{SD}, 42.8 \pm 10.7$ vs $48.8 \pm 10.4 \mathrm{~cm} / \mathrm{s}$; $p=0.040)$ and peak E velocity-to-peak A velocity ratios $(0.88 \pm 0.3$ vs $1.1 \pm 0.3 ; p=0.002)$ were also lower in DM2 patients compared with control subjects. DM2 patients with coronary artery calcification showed a lower PFR normalized to stroke volume (SV) (mean $\pm \mathrm{SD}$, $4.4 \pm 1.0$ vs $5.3 \pm 1.4$, respectively; $p=0.038)$ and lower mitral peak E velocities $(40.1 \pm 11.3$ vs $48.0 \pm 7.3 \mathrm{~cm} / \mathrm{s} ; p=0.024)$ than DM2 patients without coronary calcification. PFR normalized to SV was independently associated with the presence of coronary artery calcification $(\beta=-1.5, p=0.005)$.

CONCLUSION. DM2 decreases cardiovascular MRI-derived parameters of LV diastolic function. Patients with DM2 and coronary atherosclerosis show a more impaired LV diastolic function than patients without coronary atherosclerosis.

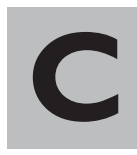

ardiovascular disease is the most frequent cause of death and disability in patients with type 2 diabetes mellitus (DM2).

DM2 is responsible for diverse cardiovascular complications such as increased coronary atherosclerosis and left ventricular (LV) dysfunction. LV diastolic dysfunction is highly prevalent in DM2 $[1,2]$, representing the earliest preclinical manifestation of LV dysfunction in patients with this condition [3-6].

Over the past decade, cardiac MRI has been widely accepted as the reference standard for the assessment of cardiac structure and function because of its high spatial and temporal resolution, excellent image quality, and lack of geometric assumptions [7]. Cardiac MRI offers a variety of alternative approaches for evaluating diastolic function $[8,9]$.

The presence of coronary artery calcium (CAC) is indicative of coronary atheroscle- rosis [10], which can be detected noninvasively by MDCT.

Coronary artery disease is commonly cited as a mechanism underlying diastolic dysfunction [11]. Many of the same factors that contribute to atherosclerosis may also produce LV diastolic dysfunction by either direct mechanisms (e.g., hypertension and age-related vascular stiffening) or secondarily via coronary artery disease progression and resulting changes in myocardial compliance [11].

There are limited and controversial published data on the relationship of CAC to LV diastolic function. Edvardsen et al. [12] reported that coronary atherosclerosis is associated with depressed regional LV systolic and diastolic wall strain measured by MRI tagging. Colletti et al. [13] found that a CAC score greater than 100 predicts an increased likelihood of clinically unsuspected LV regional wall motion abnormalities, which are 


\section{Graça et al.}

associated with lower LV ejection fractions (LVEFs) and peak filling rates (PFRs), as assessed with cardiac MRI. The results of a study by Eleid et al. [14] pointed to different conclusions. Their results in asymptomatic patients with a normal LVEF and negative findings on a cardiac stress test showed that CAC does not correlate with LV diastolic function as defined by established Doppler echocardiographic criteria.

Although all of these studies included patients with DM2, none specifically studied this population. Accordingly, the purpose of this study was to compare cardiac MRI-derived parameters of diastolic function between well-controlled uncomplicated DM2 and normoglycemic control subjects and to evaluate whether these cardiac MRI-derived parameters of LV diastolic function are related to coronary atherosclerosis.

Cardiac MRI was used to measure parameters of LV diastolic function, and the presence of coronary atherosclerosis was assessed by means of CAC scoring.

We hypothesized that in asymptomatic diabetic patients diastolic function as measured by mitral flow velocities and PFR will be reduced with the presence of coronary atherosclerosis. We also hypothesized that asymptomatic diabetic patients will have reduced diastolic function compared with normoglycemic control subjects.

\section{Subjects and Methods \\ Study Population}

We prospectively enrolled 41 subjects (21 women and 20 men) with DM2 referred to the outpatient clinical Department of Diabetology of our institution and 21 age- and sex-matched normoglycemic control subjects (nine women and 12 men) recruited from the community.

Subjects were included if they met the following inclusion criteria: age between 45 and 75 years, no symptoms or history of overt heart disease (cardiomyopathy, coronary artery disease, or valvular heart disease), no signs or symptoms of cerebrovascular disease, no abnormal findings on routine clinical and physical examinations, and normal ECG results.

Exclusion criteria were an LVEF of less than 55\%, regional LV wall motion abnormalities, and valvular heart diseases assessed by cardiovascular MRI; severe renal failure; claustrophobia; cardiovascular MR images unsuitable for quantification; type 1 diabetes mellitus; severely uncontrolled diabetes mellitus (defined as glycated hemoglobin $>12 \%$ ); and uncontrolled blood pressure at rest (defined as systolic blood pressure $>180 \mathrm{~mm} \mathrm{Hg}$ or diastolic blood pres-

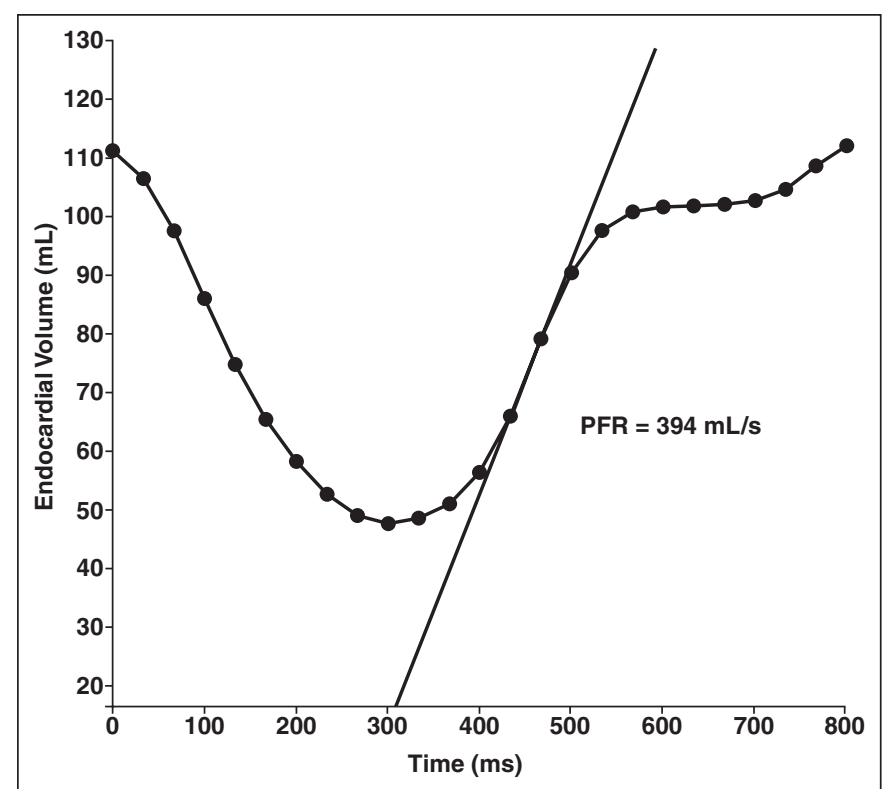

Fig. 1-48-year-old normoglycemic man. Left ventricular (LV) filling volume versus time curve and its first derivative, peak filling rate (PFR) curve, are obtained after endocardial delineation of all LV short-axis slices across all temporal phases. PFR is steepest tangent (line) to first part of filling curve and represents most rapid ventricular filling. PFR value obtained was 394 $\mathrm{mL} / \mathrm{s}$.

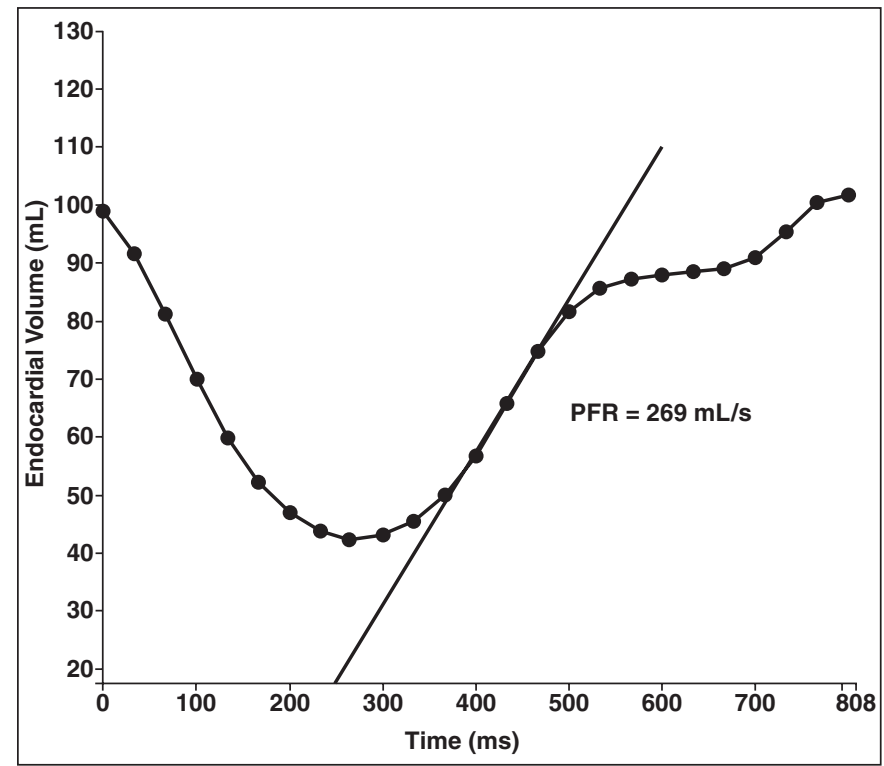

Fig. 2-45-year-old man with type 2 diabetes mellitus. Left ventricular filling volume versus time curve and peak filling rate (PRF) obtained (269 $\mathrm{mL} / \mathrm{s}$ ) are shown. PFR is steepest tangent (line) to first part of filling curve and represents most rapid ventricular filling. sure $>100 \mathrm{~mm} \mathrm{Hg}$ ). Subjects with contraindications to cardiovascular MRI were not enrolled.

The study was approved by our institutional ethics committee. Each subject gave written informed consent.

\section{Coronary Artery Calcium Score, Data \\ Acquisition, and Analysis}

CAC scoring was performed only in the DM2 group. For this purpose, unenhanced low-dose ECG-gated scanning using a 64-MDCT scanner (LightSpeed VCT XT, GE Healthcare) was performed with prospective triggering at $75 \%$ of the $\mathrm{R}-\mathrm{R}$ interval. All examinations included the entire coronary tree and were performed with the patient breath-holding in inspiration. The scanning param- eters were as follows: FOV, $25 \mathrm{~cm}$; slice thickness, $2.5 \mathrm{~mm}$; gantry rotation time, 0.35 second; tube current, 100-400 mA; and tube voltage, $120 \mathrm{kV}$.

The CAC score was determined by an experienced observer using dedicated software (SmartScore version 4.0, GE Healthcare). The total CAC score was calculated for each patient using the Agatston method [15]. For the final analysis, patients were categorized in one of two groups: group 1, CAC score greater than 0 ; or group 2, CAC score equal to 0 .

\section{Cardiac MRI Protocol}

Cardiac MRI was performed using a 3-T unit (Magnetom Trio, Siemens Healthcare). Cardiac cine images were acquired using steady-state free- 
precession sequences with retrospective ECG gating [16]. Participants were imaged in the supine position and performed a breath-hold at end expiration for each image acquisition to eliminate respiratory motion artifacts. After scout images were obtained, cine imaging was performed in four-chamber, three-chamber, and two-chamber long- and short-axis views with the use of the following parameters: 8 -mm-thick sections with a 2-mm gap between sections; TR/TE, 59.04/1.45; number of segments, 18 ; flip angle, $50^{\circ}$; matrix, $256 \times 156$; pixel size, $2.1 \times 1.6 \mathrm{~mm}$; acquired temporal resolution, $25-40 \mathrm{~ms}$; and number of reconstructed cardiac phases, 25.

A breath-held retrospectively vector ECG-gated 2D flow-sensitive phase-contrast gradient-echo sequence was used for velocity-encoded MRI flow measurements perpendicular to the orifice of the mitral valve. Velocity-encoded MRI slices were positioned in early diastole at the tip of the mitral valve leaflets. Typical imaging parameters of velocity-encoded MRI were as follows: slice thickness, $6 \mathrm{~mm}$; TR/TE, 35.6/2.33; number of segments, 3; acquired temporal resolution, 24-56 ms; flip angle, $20^{\circ} ; 25$ calculated phases; and pixel spacing, $4.5 \times 3.1 \mathrm{~mm}$. The encoding velocity was set to $130 \mathrm{~cm} / \mathrm{s}$ [9].

TABLE I: Characteristics of the Study Population

\begin{tabular}{|c|c|c|c|}
\hline Characteristics & DM2 Group ( $n=41)$ & Control Subjects ( $n=21)$ & $p$ \\
\hline Age $(y)$, mean $\pm S D$ & $58.2 \pm 7.2$ & $54.9 \pm 6.5$ & 0.078 \\
\hline Sex, no. $(\%)$ of subjects & & & 0.533 \\
\hline Male & $20(49)$ & $12(57)$ & \\
\hline Female & $21(51)$ & $9(43)$ & \\
\hline $\mathrm{BSA}\left(\mathrm{m}^{2}\right)$, mean $\pm \mathrm{SD}$ & $1.85 \pm 0.21$ & $1.80 \pm 0.16$ & 0.359 \\
\hline $\mathrm{BMI}\left(\mathrm{kg} / \mathrm{m}^{2}\right)$, mean $\pm \mathrm{SD}$ & $29.3 \pm 4.6$ & $25.0 \pm 1.7$ & $<0.001$ \\
\hline Diabetes duration (y), mean $\pm S D$ & $13.3 \pm 8.9$ & - & \\
\hline Hypertension ${ }^{\mathrm{a}}$, no. (\%) of subjects & $30(73)$ & $6(29)$ & 0.001 \\
\hline $\mathrm{LV}$ EDV $(\mathrm{mL})$, mean $\pm \mathrm{SD}$ & $96.3 \pm 20.8$ & $118.7 \pm 25.1$ & 0.001 \\
\hline LV EDV/BSA $\left(\mathrm{mL} / \mathrm{m}^{2}\right)$, mean \pm SD & $52.2 \pm 10.9$ & $65.6 \pm 11.4$ & $<0.001$ \\
\hline LV SV $(m L)$, mean \pm SD & $63.7 \pm 14.5$ & $79.9 \pm 14.4$ & $<0.001$ \\
\hline $\operatorname{LVEF}(\%)$, mean \pm SD & $66.7 \pm 8.0$ & $67.9 \pm 5.3$ & 0.485 \\
\hline $\mathrm{LV}$ mass $/ \mathrm{BSA}\left(\mathrm{g} / \mathrm{m}^{2}\right)$, mean $\pm \mathrm{SD}$ & $68.2 \pm 13.6$ & $65.6 \pm 10.0$ & 0.474 \\
\hline LV PFR $(\mathrm{mL} / \mathrm{s})$, mean $\pm \mathrm{SD}$ & $293.2 \pm 51.7$ & $375.7 \pm 102.8$ & $<0.001$ \\
\hline LV PFR/EDV $(\mathrm{mL} / \mathrm{s})$, mean \pm SD & $3.1 \pm 0.7$ & $3.2 \pm 0.8$ & 0.616 \\
\hline LV PFR/SV (mL/s), mean \pm SD & $4.7 \pm 1.1$ & $4.7 \pm 1.0$ & 0.979 \\
\hline Mitral peak E velocity $(\mathrm{cm} / \mathrm{s})$, mean \pm SD & $42.8 \pm 10.7$ & $48.8 \pm 10.4$ & 0.040 \\
\hline Mitral peak A velocity $(\mathrm{cm} / \mathrm{s})$, mean \pm SD & $49.8 \pm 11.2$ & $45.0 \pm 8.6$ & 0.069 \\
\hline Mitral E/A ratio, mean \pm SD & $0.88 \pm 0.3$ & $1.1 \pm 0.3$ & 0.002 \\
\hline
\end{tabular}

Note-Dash (-) indicates not applicable. DM2 = type 2 diabetes mellitus, BSA = body surface area, BMI = body mass index, LV = left ventricular, EDV = end-diastolic volume, SV = stroke volume, LVEF = left ventricular ejection fraction, $\mathrm{PFR}=$ peak filling rate, E/A ratio = peak E velocity-to-peak $\mathrm{A}$ velocity ratio.

ablood pressure $>140 / 90 \mathrm{~mm} \mathrm{Hg}$ or treatment with antihypertensive medication.
Analysis of the Transmitral Flow

In the flow-sensitive sequence, a round region of interest with a minimum size of $1 \mathrm{~cm}^{2}$ was placed at the center of the mitral valve orifice on a diastolic image and propagated to other phases to obtain the transmitral curve [9]. From the analysis of the transmitral flow curve, the following measurements were performed: early phase of ventricular filling (E) and atrial phase of ventricular filling (A) mean peak velocities (in centimeters per second) and peak E velocity-to-peak A velocity (E/A) ratio

\section{Statistical Analysis}

All continuous variables were tested for normal distribution. All normally distributed data are expressed as means \pm SDs. Categoric variables are expressed as counts and percentages. Betweengroup differences of the average were compared using the unpaired Student $t$ test for parametric data and the Mann-Whitney $U$ test for nonparametric data. Between-group differences in numbers and percentages were compared using the chi-square test (Fisher exact test).

The distribution of CAC scores was skewed; therefore, medians and ranges are reported.

Logarithmic transformation of CAC scoresthat is, log (total CAC score + 1) -was used for parametric evaluation.

The Pearson correlation test was used for sets of normal variables. Independent associations between coronary artery calcification and LV diastolic values were studied using a linear regression with multivariable adjustments for potentially confounding factors (e.g., age, sex, history of hypertension, body mass index [BMI], duration of diabetes, LVEF, and LV mass index).

All $p$ values of $<0.05$ were considered statistically significant. All computations were performed using software (SPSS, version 20.0, SPSS).

\section{Results}

Forty-one DM2 patients and 21 age- and sex-matched normoglycemic control subjects were studied. Demographic, clinical, and imaging data of the patients and control subjects are presented in Table 1 . The mean age was $57.2 \pm 7.1$ years (age range, $45-72$ years) and $32(52 \%)$ patients were male.

As expected, patients with DM2 had higher BMI $(p<0.001)$ than control subjects. Our population of DM2 patients had a higher prevalence of systemic arterial hypertension ( $p=0.001)$ compared with control subjects.

\section{Left Ventricular Function}

Patients with DM2 had normal conventional parameters of systolic function includ- 


\section{Graça et al.}

ing LVEF and a normal LV mass index compared with control subjects.

The absolute values of PFR were significantly higher in the control subjects (Fig. 1) than in the DM2 patients (Fig. 2) $(p<0.001)$.

The values of PFR indexed to end-diastolic volume (EDV) and stroke volume (SV) in DM2 patients and control subjects were similar ( $p=0.616, p=0.979$, respectively).

An analysis of the transmitral parameters showed lower mitral peak E velocities ( $p=$ $0.040)$ and lower E/A ratios $(p=0.002)$ in DM2 patients compared with control subjects. Mitral peak A velocities were higher in the DM2 group, although not significantly so $(p=0.069)$.

To further address the relationship between hypertension and diastolic dysfunction in the patient population, we performed a subgroup analysis of the parameters of diastolic dysfunction in the DM2 group according to the presence or absence of hypertension. The results obtained are presented in Table 2.

In this subgroup comparison, all parameters of LV diastolic function were similar in both groups.

\section{Coronary Atherosclerosis}

In the DM2 group, the average CAC score was $344 \pm 754(\mathrm{SD})$ (range, 0-4697). CAC

TABLE 2: Left Ventricular (LV) Diastolic Function Parameters in the Type 2 Diabetes Mellitus (DM2) Group According to the Presence or Absence of Systemic Arterial Hypertension

\begin{tabular}{l|c|c|c}
\hline \multicolumn{1}{c|}{ Parameters } & $\begin{array}{c}\text { Group With DM2 Only } \\
(n=11), \text { Mean } \pm \text { SD }\end{array}$ & $\begin{array}{c}\text { Group With DM2 and Systemic } \\
\text { Arterial Hypertension }(n=30), \\
\text { Mean } \pm \text { SD }\end{array}$ & $p$ \\
\hline LV PFR (mL/s) & $293.0 \pm 52.6$ & $292.1 \pm 52.7$ & 0.964 \\
LV PFR/EDV (mL/s) & $3.0 \pm 0.8$ & $3.2 \pm 0.7$ & 0.356 \\
LV PFR/SV (mL/s) & $4.5 \pm 1.1$ & $4.8 \pm 1.2$ & 0.454 \\
Mitral peak E velocity (cm/s) & $43.0 \pm 9.4$ & $42.8 \pm 11.4$ & 0.948 \\
Mitral peak A velocity (cm/s) & $50.4 \pm 6.5$ & $49.5 \pm 12.6$ & 0.774 \\
Mitral E/A ratio & $0.85 \pm 0.19$ & $0.89 \pm 0.27$ & 0.610
\end{tabular}

Note-PFR = peak filling rate, EDV = end-diastolic volume, $S V=$ stroke volume, E/A ratio = peak E velocity-topeak $A$ velocity ratio.

was absent in 14 (34\%) DM2 patients and was less than 10 in 18 (44\%) DM2 patients. Nine of $42(21 \%)$ DM2 patients had CAC scores greater than 10 .

\section{Parameters of Diastolic Function and Calcium Score}

Table 3 shows baseline and LV systolic and diastolic parameters of DM2 patients according to the presence or absence of coronary artery calcification.

DM2 patients with coronary calcification were older ( $p=0.018)$ and had longer duration of diabetes mellitus, although not signif- icantly so $(p=0.060)$. Comparing DM2 patients according to the presence or absence of coronary calcification, we found no differences in terms of sex, body surface area (BSA), BMI, or the presence of hypertension. The analysis of LV systolic function and LV mass also showed no statistically significant difference between DM2 patients with coronary calcification and those without coronary calcification.

Regarding the parameters of diastolic function, DM2 patients with coronary calcification showed lower PFR/SV (Fig. 3) $(p=0.038)$ and lower mitral peak E veloci-
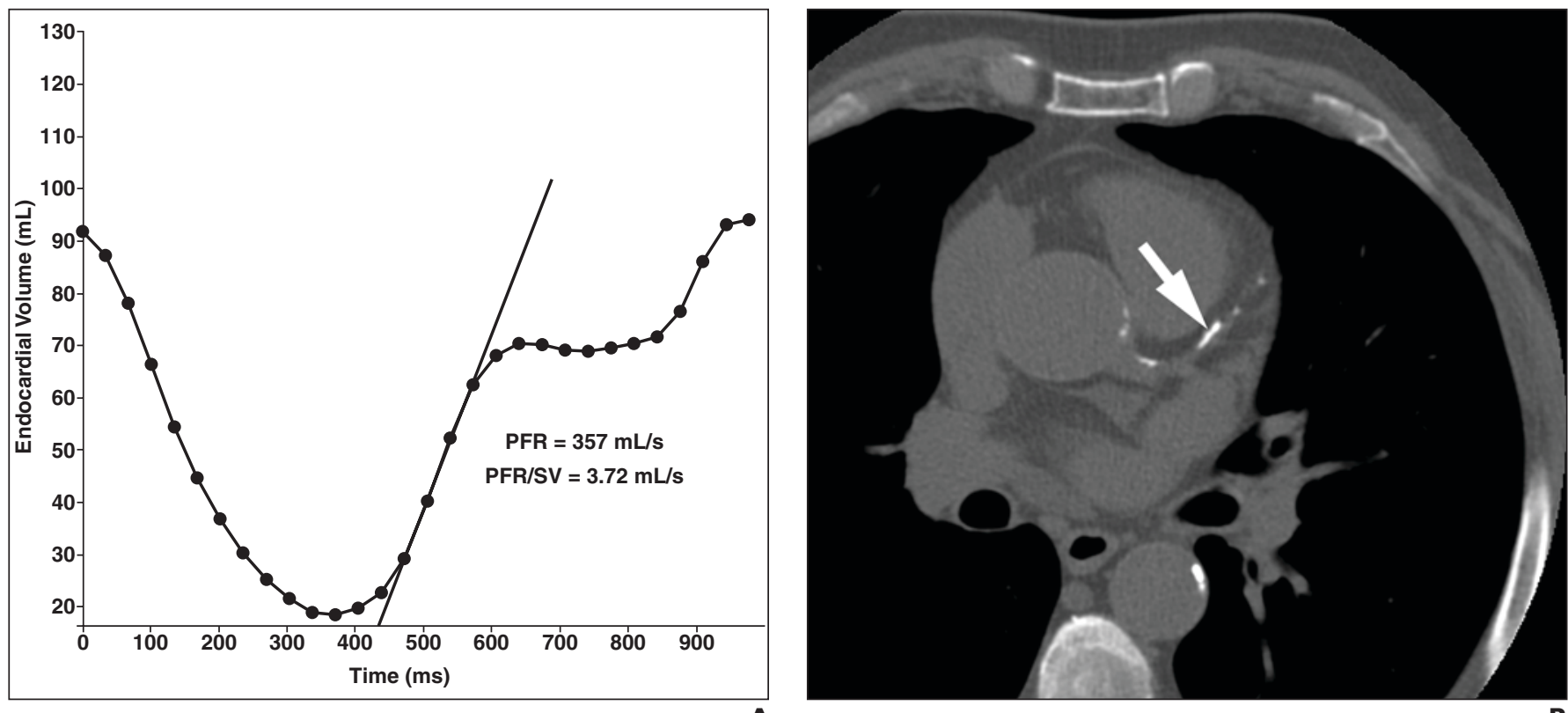

Fig. 3-67-year-old man with type 2 diabetes mellitus and coronary artery calcification.

A, Left ventricular filling volume versus time curve and peak filling rate (PFR) obtained (357 mL/s). PFR is steepest tangent (line) to first part of filling curve and represents most rapid ventricular filling. PFR indexed to stroke volume (PFR/SV) was $3.72 \mathrm{~mL} / \mathrm{s}$.

B, CT image used for coronary artery calcium scoring shows coronary artery calcifications (arrow). Agatston calcium score calculated by CT was 410. 
TABLE 3: Characteristics of Patients With Type 2 Diabetes Mellitus According to the Presence or Absence of Coronary Artery Calcification

\begin{tabular}{|c|c|c|c|}
\hline Characteristics & $\begin{array}{c}\text { Calcium Score }>0 \\
\qquad(n=27)\end{array}$ & $\begin{array}{c}\text { Calcium Score }=0 \\
\quad(n=14)\end{array}$ & $p$ \\
\hline Age $(y)$, mean \pm SD & $60.2 \pm 6.7$ & $54.6 \pm 6.8$ & 0.018 \\
\hline Sex, no. (\%) of patients & & & 0.228 \\
\hline Male & $15(56)$ & $5(36)$ & \\
\hline Female & $12(44)$ & $9(64)$ & \\
\hline $\mathrm{BSA}\left(\mathrm{m}^{2}\right)$, mean $\pm \mathrm{SD}$ & $1.86 \pm 0.17$ & $1.84 \pm 0.27$ & 0.763 \\
\hline $\mathrm{BMI}\left(\mathrm{kg} / \mathrm{m}^{2}\right)$, mean $\pm \mathrm{SD}$ & $29.1 \pm 4.5$ & $30.0 \pm 4.6$ & 0.550 \\
\hline Diabetes duration $(y)$, mean $\pm S D$ & $14.9 \pm 9.0$ & $9.4 \pm 8.4$ & 0.060 \\
\hline Hypertension ${ }^{\mathrm{a}}$, no. (\%) of patients & $19(70)$ & $11(79)$ & 0.574 \\
\hline LV EDV $(m L)$, mean \pm SD & $97.5 \pm 22.7$ & $93.9 \pm 17.1$ & 0.567 \\
\hline LV EDV/BSA $\left(\mathrm{mL} / \mathrm{m}^{2}\right)$, mean \pm SD & $52.4 \pm 10.8$ & $51.8 \pm 11.4$ & 0.863 \\
\hline LV SV $(m L)$, mean \pm SD & $65.9 \pm 15.6$ & $59.4 \pm 11.5$ & 0.139 \\
\hline $\operatorname{LVEF}(\%)$, mean \pm SD & $68.3 \pm 8.9$ & $63.7 \pm 6.6$ & 0.065 \\
\hline $\mathrm{LV}$ mass $/ \mathrm{BSA}\left(\mathrm{g} / \mathrm{m}^{2}\right)$, mean $\pm \mathrm{SD}$ & $70.9 \pm 14.3$ & $63.1 \pm 10.6$ & 0.055 \\
\hline LV PFR (mL/s), mean \pm SD & $286.6 \pm 54.2$ & $306.4 \pm 45.3$ & 0.220 \\
\hline LV PFR/EDV (mL/s), mean \pm SD & $3.0 \pm 0.7$ & $3.4 \pm 0.8$ & 0.142 \\
\hline LV PFR/SV (mL/s), mean \pm SD & $4.4 \pm 1.0$ & $5.3 \pm 1.4$ & 0.038 \\
\hline Mitral peak E velocity $(\mathrm{cm} / \mathrm{s})$, mean $\pm S D$ & $40.1 \pm 11.3$ & $48.0 \pm 7.3$ & 0.024 \\
\hline Mitral peak A velocity $(\mathrm{cm} / \mathrm{s})$, mean \pm SD & $49.8 \pm 13.2$ & $49.8 \pm 6.2$ & 0.997 \\
\hline Mitral E/A ratio, mean \pm SD & $0.83 \pm 0.3$ & $0.96 \pm 0.2$ & 0.086 \\
\hline
\end{tabular}

Note-BSA = body surface area, $\mathrm{BMI}=$ body mass index, $\mathrm{LV}=$ left ventricular, EDV = end-diastolic volume, SV = stroke volume, $\mathrm{LVEF}=$ left ventricular ejection fraction, $\mathrm{PFR}=$ peak filling rate, $\mathrm{E} / \mathrm{A}$ ratio = peak $\mathrm{E}$ velocity-topeak A velocity ratio.

aBlood pressure $>140 / 90 \mathrm{~mm} \mathrm{Hg}$ or treatment with antihypertensive medication. ties $(p=0.024)$ compared with DM2 patients without coronary calcification (Fig. 4).

Univariable and Multivariable Analyses

In the DM2 group, an increasing CAC score was negatively correlated with PFR/SV $(r=-0.68, p<0.001)$ (Fig. 5).

By multivariable analysis, PFR/SV was independently associated with the presence of coronary calcification $(\beta=-1.5, p=0.005)$ after adjustment for age, sex, BMI, presence of hypertension, diabetes duration, LVEF, and LV mass index.

\section{Discussion}

In this study, CAC score and parameters of diastolic function in patients with DM2 were investigated. There were two major observations. First, differences in diastolic function were observed between DM2 patients and control subjects-in particular, in LV PFR values, mitral peak $\mathrm{E}$ velocities, and $\mathrm{E} / \mathrm{A}$ ratios. Second, the parameters of diastolic function were different between DM2 patients with coronary calcification and those without coronary calcification - in particular, LV $\mathrm{PFR} / \mathrm{SV}$ values and mitral peak E velocities.

Differences in Left Ventricular Diastolic Measures

LV filling profiles have been used to assess diastolic function by other imaging investigations, such as radionuclide cineangi-
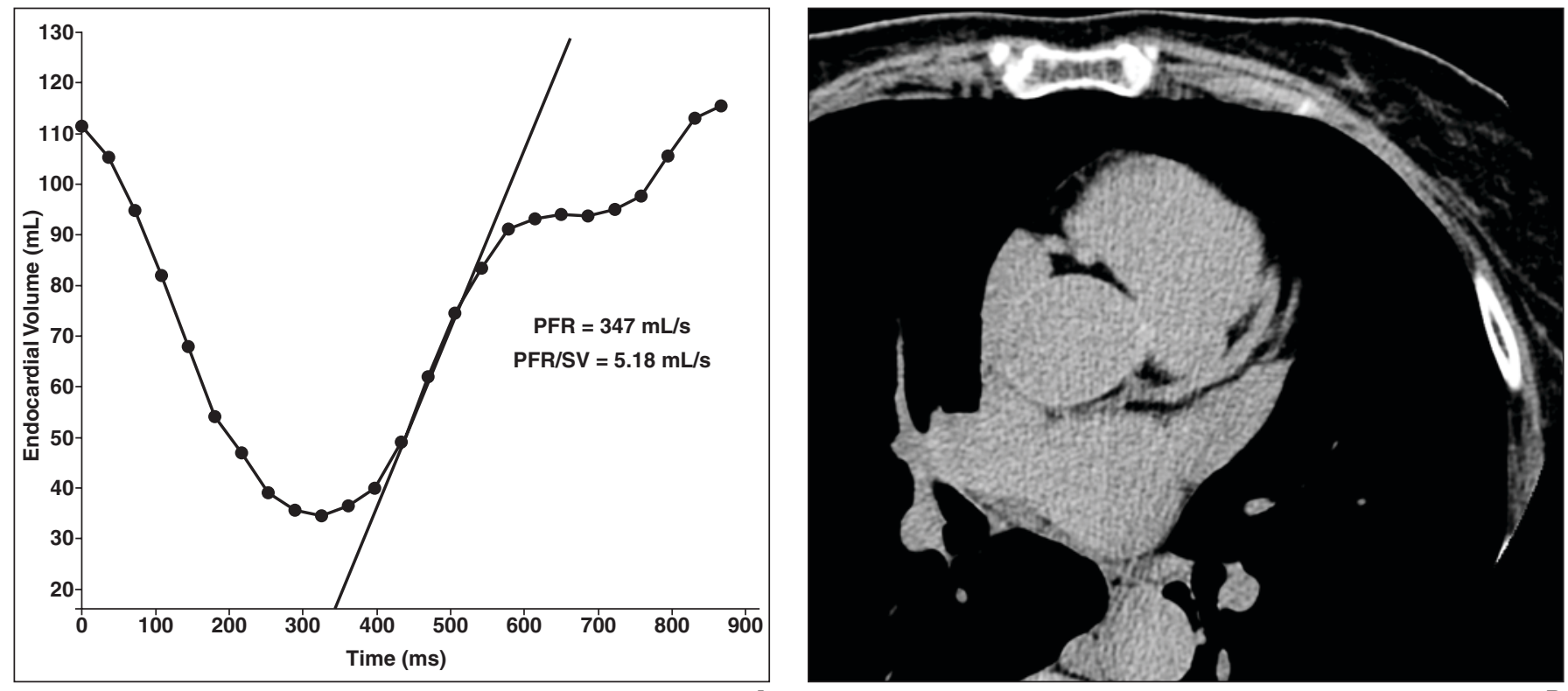

A

Fig. 4-55-year-old woman with type 2 diabetes mellitus and no coronary artery calcification.

A, Left ventricular filling volume versus time curve and peak filling rate (PFR) obtained ( $347 \mathrm{~mL} / \mathrm{s}$ ). PFR is steepest tangent (line) to first part of filling curve and represents most rapid ventricular filling. PFR indexed to stroke volume (PFR/SV) was $5.18 \mathrm{~mL} / \mathrm{s}$.

B, CT image used for coronary artery calcium scoring shows no coronary artery calcifications. Agatston calcium score calculated by CT was 0. 


\section{Graça et al.}

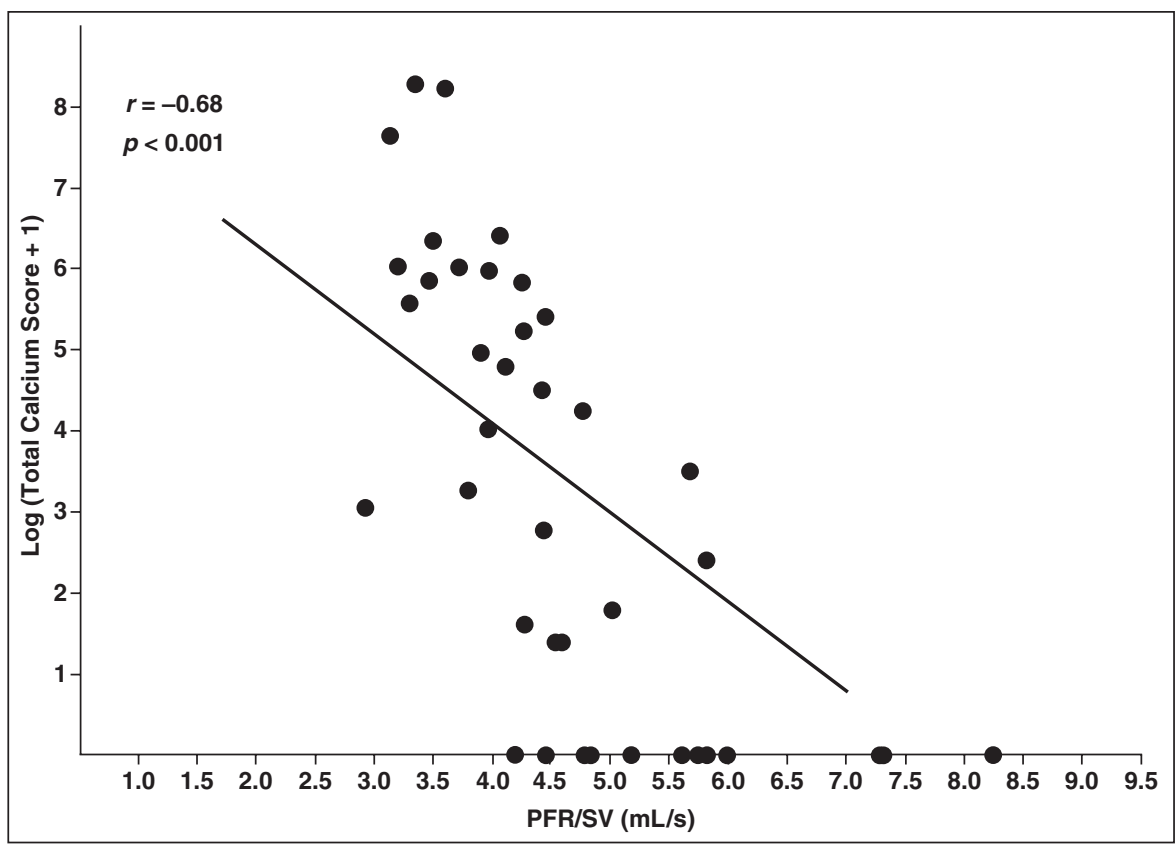

Fig. 5-Scatterplot and linear fit of relationship of peak filling rate (PFR) indexed to stroke volume (PFR/SV) with coronary artery calcium (CAC) score.

ography and SPECT. Similar applications for cardiac MRI have been impractical because manual planimetry of all LV images across all temporal phases would typically require tracing of more than 200 images per patient. With improvements in postprocessing tools, it is now possible to semiautomatically segment all phases and quickly provide the time-varying course of the LV volume during the cardiac cycle. The ventricular relaxation abnormalities can be diagnosed by a low PFR. This evaluation is based on conventional short-axis sequences without the necessity to perform a dedicated acquisition. The use of PFR values indexed to EDV and SV minimizes the dependency of PFR on EDV and heart rate. We found that the LV PFR can be easily obtained as an addition to the assessment of LV systolic function with semiautomatic contour detection and it may become a valuable asset in the evaluation of LV diastolic function.

In this study, parameters of LV diastolic function were impaired in DM2 patients compared with normoglycemic control subjects. The mitral peak E velocity, E/A ratio, and LV PFR were lower in DM2 patients, indicating impaired myocardial relaxation and increased myocardial stiffness, which are the hallmarks of diastolic dysfunction [18]. DM2-related changes in LV diastolic properties are well recognized with a decrease in early diastolic filling, directly influenced by abnormal LV relaxation, mainly due to a derangement in energy supply and an increased passive stiffness due to remodeling [19].

We acknowledge that coexisting arterial hypertension augments the impairment of LV diastolic function in diabetic patients and may partially explain the changes reported.

Interestingly, Di Bonito et al. [20] observed diastolic dysfunction in 16 normotensive patients with DM2 without microvascular complications and a short disease duration $(<1$ year). Likewise, previous studies have shown that more than $40-75 \%$ of normotensive diabetic patients under excellent glycemic control had diastolic dysfunction $[1,2,21]$. In our study, the parameters of diastolic function were not different in the patients with DM2 and hypertension compared with the DM2 patients without hypertension.

\section{Diastolic Function and Coronary Atherosclerosis}

Our study of DM2 patients shows an association between diastolic function assessed by cardiac MRI and coronary artery calcification assessed by CT.

In our cohort of DM2 patients, lower PFR/SV and lower mitral peak E values were significantly associated with coronary calcification. Furthermore, the presence of coronary calcification was independently associated with PFR/SV, increasing the strength of this association. Our results are consistent with the findings of other studies. Colletti et al. [13] studied 386 subjects, including 39 patients with diabetes mellitus, and also found a relation of a CAC score of greater than 100 with decreased MRI-derived parameters of LV diastolic filling. Scholte et al. [22] reported similar conclusions using echocardiography-derived indexes of diastolic function (transmitral and pulmonary vein flow recording) and systolic function (global longitudinal strain). Their results point to an association of coronary atherosclerosis with subclinical LV dysfunction evaluated in DM2 patients [22].

Mechanisms underlying the relationship of subclinical atherosclerosis and diastolic function are probably related to vascular function.

Poulsen et al. [23] suggested that abnormal LV filling in DM2 patients is closely associated with abnormal myocardial perfusion on myocardial perfusion scintigraphy. Macrovascular coronary artery disease could also contribute to this subclinical myocardial damage [22]. In coronary macrovasculature, formation of atheroma may lead to luminal obliteration, recurrent thrombosis, distal embolization, and clinically silent microinfarctions [11].

The findings of this study contribute to the available knowledge, favoring the hypothesis that there is a link between subclinical LV diastolic dysfunction and asymptomatic atherosclerosis.

In our study, the DM2 patients with coronary artery calcification had greater LV mass than the DM2 patients without coronary calcification, but the difference did not quite achieve acceptable levels of statistical significance $(p=0.055)$. LV mass represents an endpoint of cumulative LV insult, and although not a diagnostic criteria for LV diastolic dysfunction, increased LV mass is associated with diastolic dysfunction ranging from abnormal relaxation to coexistent restrictive physiology due to increased myocardial stiffness [24]. Systemic arterial hypertension and diabetes mellitus are known causes of LV hypertrophy. Notwithstanding the high prevalence of arterial hypertension in our population, none of our subjects had an LV mass above the normal range (LV mass indexed to BSA $>86 \mathrm{~g} / \mathrm{m}^{2}$ for men and $>72 \mathrm{~g} / \mathrm{m}^{2}$ for women) $[25,26]$.

The originality of our study is the use of cardiac MRI-derived parameters of diastolic function and the relatively high number of DM2 patients with a low CAC score. We had a relatively large percentage of DM2 patients 
with a CAC score equal to zero, something that we were not expecting in a population of DM2 patients between the ages of 45 and 72 years.

A basic limitation of this study must be acknowledged. This study was a cross-sectional study with a relatively small number of subjects; therefore, studies using larger patient cohorts are needed to clarify the association between LV diastolic function and coronary atherosclerosis.

Our findings are limited by an inability to eliminate causal relationships with important clinical factors, such as medications used, serum concentrations of glucose and glycosylated hemoglobin (HbA1c), dyslipidemia, retinopathy, microalbuminuria, and smoking status. Also, the high prevalence of hypertension in our population makes it difficult to interpret the findings and clarify the responsibility of diabetes in the results obtained.

The possibility of influence of myocardial ischemia on LV diastolic function cannot be completely excluded. The evaluation of coronary stenosis and plaque characterization were not performed in our study because CT angiography is not recommended in asymptomatic DM2 patients. Because myocardial perfusion, endothelial function, coronary flow reserve, and arterial stiffness were not evaluated in the current study, a more comprehensive understanding of the pathophysiologic process should be the focus of future research.

We consider the analysis of cine and transmitral flow images to be sufficient to exclude left-sided valvular disease as a potential pitfall of abnormal transmitral flow. Regarding the exclusion of infiltrative myocardial disease, we neither studied myocardial delayed enhancement nor performed endomyocardial biopsy. Nevertheless, none of our subjects had increased LV myocardial end-diastolic thickness, pericardial thickening, pericardial effusion, or pleural effusion.

Recent findings [27] have shown an improvement in transmitral flow assessment by applying 3D three-directional velocity-encoding instead of the conventional approach of $2 \mathrm{D}$ one-directional through-plane velocity-encoded MRI with a fixed acquisition plane used in our study.

Normalizing early mitral velocity (E) for the influence of myocardial relaxation by combining E with early diastolic mitral septal tissue velocity (Ea) may be performed by MR tissue phase-contrast imaging, and this is an important parameter of diastolic function that has been established [28]. On the other hand, there are still conflicting data regarding the relation of E/Ea ratio and LV filling pressure $[29,30]$.

The evaluation of coronary stenosis and characterization of plaque were not performed in our study because CT angiography is not recommended in asymptomatic DM2 patients

This study highlights certain key points for the routine use of cardiac MRI to study diastolic function in diabetic patients and the relation of diastolic function with coronary atherosclerosis. First, cardiac MRI shows a decrease in diastolic function in DM2 and may allow the diagnosis of subclinical diastolic dysfunction in this high-risk population. Second, diastolic dysfunction is possibly related to the presence of coronary atherosclerosis.

\section{References}

1. Boyer JK, Thanigaraj S, Schechtman KB, Pérez JE. Prevalence of ventricular diastolic dysfunction in asymptomatic, normotensive patients with diabetes mellitus. Am J Cardiol 2004; 93:870-875

2. Zabalgoitia M, Ismaeil MF, Anderson L, Maklady FA. Prevalence of diastolic dysfunction in normotensive, asymptomatic patients with well-controlled type 2 diabetes mellitus. Am J Cardiol 2001; 87:320-323

3. Celentano A, Vaccaro O, Tammaro P, et al. Early abnormalities of cardiac function in non-insulindependent diabetes mellitus and impaired glucose tolerance. Am J Cardiol 1995; 76:1173-1176

4. Galderisi M. Diastolic dysfunction and diabetic cardiomyopathy: evaluation by Doppler echocardiography. J Am Coll Cardiol 2006; 48:1548-1551

5. Di Bonito P, Moio N, Cavuto L, et al. Early detection of diabetic cardiomyopathy: usefulness of tissue Doppler imaging. Diabet Med 2005; 22:1720-1725

6. Poirier P, Bogaty P, Garneau C, Marois L, Dumesnil JG. Diastolic dysfunction in normotensive men with well-controlled type 2 diabetes: importance of maneuvers in echocardiographic screening for preclinical diabetic cardiomyopathy. Diabetes Care 2001; 24:5-10

7. Grothues F, Smith GC, Moon JC, et al. Comparison of interstudy reproducibility of cardiovascular magnetic resonance with two-dimensional echocardiography in normal subjects and in patients with heart failure or left ventricular hypertrophy. Am J Cardiol 2002; 90:29-34

8. Rathi VK, Doyle M, Yamrozik J, et al. Routine evaluation of left ventricular diastolic function by cardiovascular magnetic resonance: a practical approach. J Cardiovasc Magn Reson 2008; 10:36

9. Caudron J, Fares J, Bauer F, Dacher JN. Evaluation of left ventricular diastolic function with cardiac MR imaging. RadioGraphics 2011; 31:239-259
10. Schmermund A, Baumgart D, Görge G, et al. Measuring the effect of risk factors on coronary atherosclerosis: coronary calcium score versus angiographic disease severity. J Am Coll Cardiol 1998; 31:1267-1273

11. Ohara T, Little WC. Evolving focus on diastolic dysfunction in patients with coronary artery disease. Curr Opin Cardiol 2010; 25:613-621

12. Edvardsen T, Rosen BD, Pan L, et al. Regional diastolic dysfunction in individuals with left ventricular hypertrophy measured by tagged magnetic resonance imaging: the Multi-Ethnic Study of Atherosclerosis (MESA). Am Heart J 2006; 151:109-114

13. Colletti PM, Dustin LD, Wong ND, et al. Does coronary calcium score predict future cardiac function? Association of subclinical atherosclerosis with left ventricular systolic and diastolic dysfunction at MR imaging in an elderly cohort. $\mathrm{Ra}$ diology 2010; 257:64-70

14. Eleid MF, Appleton CP, Lopez AG, Cha S, Hurst RT. Coronary artery plaque burden does not affect left ventricular diastolic function in asymptomatic adults with normal ejection fraction. $J A m$ Soc Echocardiogr 2011; 24:909-914

15. Agatston AS, Janowitz WR, Hildner FJ, Zusmer NR, Viamonte M, Detrano R. Quantification of coronary artery calcium using ultrafast computed tomography. J Am Coll Cardiol 1990; 15:827-832

16. Ginat DT, Fong MW, Tuttle DJ, Hobbs SK, Vyas RC. Cardiac imaging. Part 1 . MR pulse sequences, imaging planes, and basic anatomy. AJR 2011; 197:808-815

17. Heiberg E, Sjögren J, Ugander M, Carlsson M, Engblom H, Arheden H. Design and validation of Segment: freely available software for cardiovascular image analysis. BMC Med Imaging 2010; 10:1

18. Borlaug BA, Paulus WJ. Heart failure with preserved ejection fraction: pathophysiology, diagnosis, and treatment. Eur Heart J 2011; 32:670-679

19. von Bibra H, St. John Sutton M. Diastolic dysfunction in diabetes and the metabolic syndrome: promising potential for diagnosis and prognosis. Diabetologia 2010; 53:1033-1045

20. Di Bonito P, Cuomo S, Moio N, et al. Diastolic dysfunction in patients with non-insulin-dependent diabetes mellitus of short duration. Diabet Med 1996; 13:321-324

21. Masugata H, Senda S, Goda F, et al. Left ventricular diastolic dysfunction in normotensive diabetic patients in various age strata. Diabetes Res Clin Pract 2008; 79:91-96

22. Scholte AJ, Nucifora G, Delgado V, et al. Subclinical left ventricular dysfunction and coronary atherosclerosis in asymptomatic patients with type 2 diabetes. Eur J Echocardiogr 2011; 12:148-155

23. Poulsen MK, Henriksen JE, Dahl J, et al. Left ventricular diastolic function in type 2 diabetes 


\section{Graça et al.}

mellitus: prevalence and association with myocardial and vascular disease. Circ Cardiovasc Imaging 2010; 3:24-31

24. de Simone G, Kitzman DW, Palmieri V, et al. Association of inappropriate left ventricular mass with systolic and diastolic dysfunction: the HyperGEN study. Am J Hypertens 2004; 17:828-833

25. Clay S, Alfakih K, Radjenovic A, Jones T, Ridgway JP, Sinvananthan MU. Normal range of human left ventricular volumes and mass using steady state free precession MRI in the radial long axis orientation. MAGMA 2006; 19:41-45
26. Maceira AM, Prasad SK, Khan M, Pennell DJ. Normalized left ventricular systolic and diastolic function by steady state free precession cardiovascular magnetic resonance. J Cardiovasc Magn Reson 2006; 8:417-426

27. Westenberg JJ, Roes SD, Ajmone Marsan N, et al. Mitral valve and tricuspid valve blood flow: accurate quantification with 3D velocity-encoded MR imaging with retrospective valve tracking. Radiology 2008; 249:792-800

28. Nagueh SF, Appleton CP, Gillebert TC, et al. Recommendations for the evaluation of left ventricu- lar diastolic function by echocardiography. Eur $J$ Echocardiogr 2009; 10:165-193

29. Bhella PS, Pacini EL, Prasad A, et al. Echocardiographic indices do not reliably track changes in leftsided filling pressure in healthy subjects or patients with heart failure with preserved ejection fraction. Circ Cardiovasc Imaging 2011; 4:482-489

30. Kumar K, Nepomuceno RG, Chelvanathan A, et al. The role of tissue Doppler imaging in predicting left ventricular filling pressures in patients undergoing cardiac surgery: an intraoperative study. Echocardiography 2013; 30:271-278

\section{FOR YOUR INFORMATION}

This article is available for CME and Self-Assessment (SA-CME) credit that satisfies Part II requirements for maintenance of certification (MOC). To access the examination for this article, follow the prompts associated with the online version of the article. 